\title{
Evaluation of systemic inflammatory response syndrome-negative sepsis from a Chinese regional pediatric network
}

\author{
Yuanyuan Wang ${ }^{1 \dagger}$, Xiaofei Lin $^{2 \dagger}$, Hongni Yue ${ }^{2}$, Niranjan Kissoon ${ }^{3}$, Bo Sun ${ }^{1 *}$ and Collaborative Study Group for \\ Pediatric Sepsis in Huai'an
}

\begin{abstract}
Background: The identification of sepsis in children varies depending on the definition used. Our purpose was to compare clinical data and outcome of atypical sepsis, manifested as having sepsis but not fulfilling the criteria of systemic inflammatory response syndrome (SIRS-negative sepsis, SNS), in children based on the modified Angus criteria with those of sepsis (S) and severe sepsis (SS) based on the international consensus criteria.

Methods: Pediatric departments in 11 regional city and county referral hospitals with emergency and intensive care settings in Huai'an serving for 843,000 children participated in a parallel multicenter prospective survey. Clinical data registry was used to recruit those who fulfilled the diagnostic criteria for pediatric sepsis from all admissions $(n=27,836)$ from 28 days to 15 years old, from September 1, 2010 to August 31, 2011.

Results: A total of 1606 children met the criteria for pediatric sepsis and were divided into three groups: $\mathrm{S}$, ( $n=1377)$, SS ( $n=153$, including 32 septic shock), based on the consensus definition criteria, and SNS $(n=76)$ based on the modified Angus criteria. Most deaths (38/54, 70.3\%) occurred within three days of admission. The SNS mainly occurred in infants and was associated with cardiopulmonary and neurologic dysfunction without satisfying the SIRS criteria.

Conclusions: SNS differed from SS in that it predominantly affected infants and manifested with cardiopulmonary and neurologic dysfunction. There were no laboratory variables which were useful in identification of SNS, or predicting response to therapy or outcome.
\end{abstract}

Keywords: Infection, Outcome, Pediatrics, Sepsis, Systemic inflammatory response syndrome, Therapy

\section{Background}

Severe sepsis and septic shock in infancy and early childhood remain a significant cause of morbidity and mortality worldwide. The World Health Organization statistics suggests that at least $50 \%$ of death in children less than 4 years old in developing and underdeveloped countries and regions may be associated with sepsis [1-3]. Even in the developed countries, pediatric sepsis remains an important burden with regard to its incidence and outcome $[4,5]$.

\footnotetext{
* Correspondence: bsun@shmu.edu.cn

†Yuanyuan Wang and Xiaofei Lin contributed equally to this work.

'Departments of Pediatrics and Pediatric Critical Care, Children's Hospital of

Fudan University, 399 Wanyuan Road, Shanghai 201102, China

Full list of author information is available at the end of the article
}

The mortality rate of pediatric sepsis from pediatric intensive care units (PICUs) of developing countries is higher than $50 \%[6,7]$, and is related to many factors including availability and affordability of care.

Studies in China showed that the frequency of sepsis in children in PICUs was 7.4-26.2\%, and the associated mortality rate was $17-31 \%$ [8]. Moreover, among those children with acute hypoxemic respiratory failure and acute respiratory distress syndrome with sepsis as underlying pathologies had a very high mortality (>70\%), relative risk of death was 3-4 times higher than those without sepsis, and affecting mostly in those under 6 years old [9-11]. The identification of sepsis was based on the presence of systemic inflammatory

(c) The Author(s). 2019 Open Access This article is distributed under the terms of the Creative Commons Attribution 4.0 International License (http://creativecommons.org/licenses/by/4.0/), which permits unrestricted use, distribution, and reproduction in any medium, provided you give appropriate credit to the original author(s) and the source, provide a link to the Creative Commons license, and indicate if changes were made. The Creative Commons Public Domain Dedication waiver (http://creativecommons.org/publicdomain/zero/1.0/) applies to the data made available in this article, unless otherwise stated. 
response syndrome (SIRS) and presumed infection [12]. However, recently it is recognized that using the SIRS criteria one in eight cases of sepsis may be missed [13]. These cases while not satisfying SIRS criteria (SIRS negative) are identified by physicians by being infected or suspected of having an infection and organ dysfunction (the modified Angus criteria, [14]). Thus they are considered to have SIRS negative sepsis (SNS, [13]), synonymous as Angus-criteria sepsis, or atypical sepsis. While both criteria have gained acceptance, there was little data on the clinical course, laboratory data, therapy and outcome based on the criteria used for diagnosis of pediatric sepsis, severe sepsis (SS) and SNS, especially from a regional pediatric emergency service for all children's population from post-neonatal infancy.

In our previous multicenter prospective survey of 1606 cases of sepsis and severe sepsis [15], we identified a group of patients with infection who did not satisfy the international criteria for sepsis [12] but was diagnosed as having sepsis based on the modified Angus criteria [14], hence we consider these patients to be SNS. The purpose of present study was to compare these patients using the modified Angus criteria with those of sepsis and SS diagnosed using the international criteria with regard to clinical and laboratory findings, treatment and outcomes. We hypothesized that SNS may have different manifestation from but similar clinical risks to SS, and should be treated with the same strategy and particular attention.

\section{Subjects and methods \\ Study population and inclusion criteria}

This descriptive prospective, cross-sectional study was performed in Huai'an city, Jiangsu province in China, a semi-agronomic prefectural region, with 50\% rural residents of a total population of 5.4 million, which was similar to the national average level of economic development as judged by the gross domestic production, resident income, and health care levels. To ensure quality of the survey, a collaborative study group was established among pediatric departments from 10 level II and III county and city general hospitals and a women and children's hospital, of Huaian region, consisting of a total of 541 ward beds and 16 PICU beds which were located in two tertiary care hospitals. The rural residents were subjected to the New Rural Cooperative Medical Scheme, a universal health insurance policy since 2010 which enabled prompted emergency and admission to care. Details of study protocol and compliance of investigator performance are reported elsewhere [15], and the patient enrolment and eligible for data analysis are shown in Fig. 1.

This study was coordinated by Huai'an Women and Children's Hospital in collaboration with Children's Hospital of Fudan University following approval of the

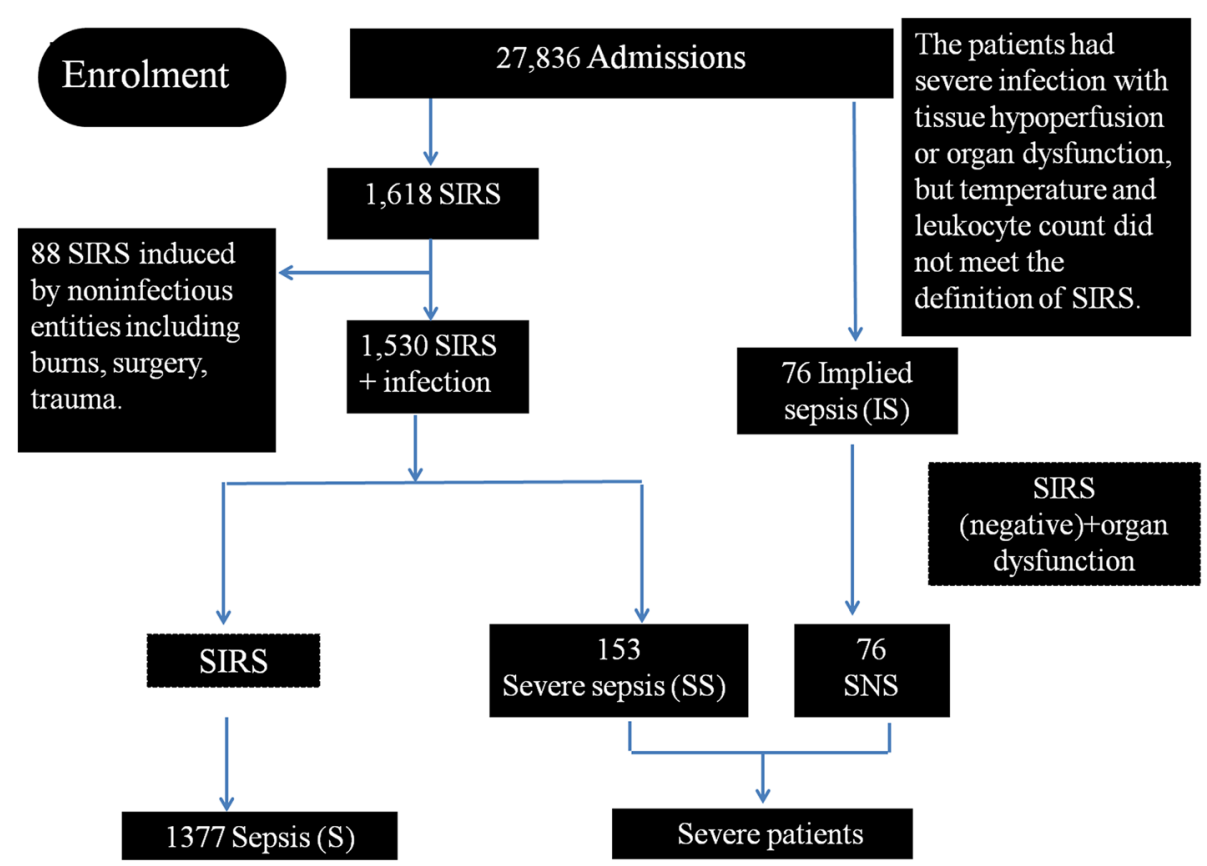

Fig. 1 Distribution of patients and groups analyzed in the Huai'an Sepsis Study. Patients initially presented as systemic inflammatory response syndrome (SIRS)-negative sepsis (SNS), or modified Angus criteria for sepsis definition (severe infection with tissue hypoperfusion or organ dysfunction, but with fewer than two criteria of conventional criteria for SIRS), S (sepsis, equal to or more than two SIRS criteria), and severe sepsis (SS, sepsis with acute respiratory distress syndrome, cardiovascular organ dysfunction, or two or more other acute organ dysfunctions), according to the worst sepsis stage reached during hospitalization 
study protocol by local ethics committees/institutional review boards [15]. To ensure quality of the study, training course for clinical staffs participating the study was provided prior to the commence of the survey, and case record form of each patient enrolled in the study was prospectively monitored by local co-investigator. At the conclusion of the treatment or in case of death, the case record form was completed, and completeness and accuracy checked by both co-investigator and staffs of the coordination center.

All children, 28 days to 15 years old, who were consecutively admitted to the participating hospitals from September 1, 2010 to August 31, 2011, were actively screened daily for the presence of sepsis (S), SS or septic shock using a diagnostic tool adopted from the international definitions for pediatric sepsis and organ dysfunction [12]. Patients were prospectively enrolled in the study when onset of S or SS was suspected based on the international guidelines and or was clinically suspected by attending physicians based on suspected or proven infection and organ dysfunction (modified Angus criteria for pediatrics) [14]. Severe sepsis was defined as sepsis with respiratory failure and/or acute respiratory distress syndrome, cardiovascular organ dysfunction, or two or more other acute organ dysfunction. Septic shock was defined as cardiovascular dysfunction or the presence of altered peripheral perfusion despite optimal fluid resuscitation [15-20]. The modified Angus criteria defined SNS as patients who does not meet the international consensus criteria but has a suspected or proven infection with at least one organ dysfunction or failure [14].

For each patient who satisfied the international guidelines for S and SS, clinical management and outcome were reported elsewhere [15]. For this study, the clinical management and outcome of children with SNS was reviewed for comparison. In addition, physical examination of peripheral perfusion, hematological and biochemical variables from peripheral blood samples were also recorded for site of infection, isolated pathogens, antibiotic therapy (prophylactic, based on resistance), and organ dysfunction/failure. These included total proteins, albumin, total bilirubin, aspartate aminotransferase, lactate dehydrogenase, serum creatinine, creatine kinase-myocardial band isoenzyme and blood routine examination during hospitalization. Values of these parameters in the first few days of study enrolment were compared among the patients in group with diagnosis of sepsis (S), SS [12, 15, 21] and SNS [14].

\section{Statistical analysis}

Statistical analysis was performed using SPSS software v. 16.0. Continuous variables are summarized as median and interquartile range (IQR) or range, or as means and standard deviation (SD). Categorical data are presented as numbers and proportions in percentage. Comparisons of continuous variables are performed with the use of analysis of variance followed by Student-Newmann-Keuls post hoc test for between-group differences, or by Student $\mathrm{t}$ test, and comparisons of categorical variables are performed with Chi square test. A $P$ value $<0.05$ was considered statistically significant.

\section{Results}

\section{Study population}

Of the 27,836 children admitted to the 11 participating hospitals during the study period, a total of 1606 (5.8\%) cases met the sepsis criteria [12]. In the end, a total of 153 SS and 76 SNS cases were included in the database. Table 1 illustrates baseline data and clinical characteristics of the three groups at study entry. Of the 1606 cases, age distribution was as follows: $57.5 \%$ in 1 month to $<2$ years, 23.3\% 2-4 years, 9.5\% 5-7 years, and 9.5\% 8-14 years old. The incidence of sepsis was the highest in children under five years old $(n=1297,80.8 \%)$ with an overall higher incidence in boys than in girls in every group. In SNS group, most $(65 / 76,85 \%)$ of cases had sepsis onset below one year old. The proportion of patients admitted to the PICU was 110 (8.0\%) of S, 116 (75.8\%) SS and 45 (59.2\%) SNS. The duration of hospital stay for all sepsis patients was $8.7 \pm 3.0$ [median (range) $8(1-38)]$ days in S, $11.2 \pm 10.6$ [10 (1-70)] days in SS and $13.2 \pm 6.7$ [12(2-36)] days in SNS. The hospital costs were $3408 \pm 2986$ [2611 (150-38,713)] Chinese Yuan $(\mathrm{CNY})$ in S, 8884 $\pm 8230[6620(136-50,763)]$ Yuan in SS and $7916 \pm 5193$ [6315 (1079-27,964)] Yuan in SNS.

\section{Clinical manifestations}

An average time interval between the onset of signs and symptoms of infection to the initial diagnosis of SS and SNS was $6.1 \pm 8.7$ (median [IQR], 3 [2-7]) and $5.4 \pm 5.1$ (4 [3-6]) days, respectively [15]. In 578 (36\%) patients who met SIRS criteria the initial abnormal temperature and/or white blood cell counts (WBC) at enrolment was transient and resolved by $48 \mathrm{~h}$ after enrolment [15]. Most of these patients received first treatment at local clinics, or received oral medication at home at the onset of symptom. In SNS group there were 36 with tachycardia, 68 high mean respiratory rate, none with core body temperature $>38.5^{\circ} \mathrm{C}$ or $<36^{\circ} \mathrm{C}$, and none with peripheral WBC significantly out of the ranges for ages. Average levels of the peripheral WBC was $15-20 \times 10^{9} / \mathrm{L}$ in S, with significant reduction from study entry to the period during $24-72 \mathrm{~h}$ of treatment whereas there was no significant changes $\left(10-15 \times 10^{9} / \mathrm{L}\right)$ for both SS and SNS groups.

Most patients in S group had mild depression of consciousness, however in SS and SNS patients, delirium, somnolence and coma were often found. Loss of consciousness and coma were associated with poor prognosis with a case 
Table 1 Baseline data and clinical characteristics of the patients at study entry and outcome

\begin{tabular}{|c|c|c|c|c|}
\hline Variables & Sepsis $^{a}$ & $S S^{a}$ & SNS & p \\
\hline Case, $n$ & 1377 & $153^{b}$ & 76 & \\
\hline Age, year, mean (median) & $3.3(2.0)$ & $1.6(0.6)$ & $0.7(0.3)$ & 0.000 \\
\hline Gender, boys (girls), $n$ (n) & $904(473)$ & $95(58)$ & $50(26)$ & 0.678 \\
\hline Rural residents, $n$ (\%) & $843(61.7)$ & $122(79.7)$ & $62(81.6)$ & 0.000 \\
\hline Temperature, mean $\pm \mathrm{SD},{ }^{\circ} \mathrm{C}$ & $39.0 \pm 0.7$ & $38.3 \pm 1.4$ & $37.2 \pm 0.6$ & 0.000 \\
\hline Underlying diseases, $n$ (\%) & $55(4.0)$ & $55(35.9)$ & $22(28.9)$ & 0.000 \\
\hline C-reactive protein (CRP) & $36.9 \pm 55.0$ & $32.5 \pm 58.5$ & $6.7 \pm 18.5$ & 0.000 \\
\hline \multicolumn{5}{|l|}{ Sources of infection, $n(\%)$} \\
\hline Lower respiratory tract & $418(30.4)$ & $98(64.0)$ & $68(89.5)$ & 0.000 \\
\hline Upper respiratory tract & $414(30.1)$ & 0 & 0 & 0.000 \\
\hline Central nervous system & $145(10.5)$ & $20(13.1)$ & $5(6.6)$ & 0.000 \\
\hline Unknown & $265(19.2)$ & $13(7.4)$ & $1(1.3)$ & 0.000 \\
\hline Gastrointestinal system & $67(4.9)$ & $17(11.1)$ & $1(1.3)$ & 0.000 \\
\hline Blood stream & $49(3.6)$ & $4(2.6)$ & $1(1.3)$ & 0.000 \\
\hline Soft tissue & $18(1.3)$ & $1(0.7)$ & 0 & 0.000 \\
\hline \multicolumn{5}{|l|}{ Mental status, $n(\%)$} \\
\hline Irritability & $21(1.5)$ & $34(22.2)$ & $21(27.6)$ & 0.000 \\
\hline Convulsions & $147(10.7)$ & $32(20.9)$ & $5(6.6)$ & 0.000 \\
\hline Twitches & $6(0.4)$ & $3(2.0)$ & 0 & 0.000 \\
\hline Decreased level of consciousness & $275(20.0)$ & $121(79.1)$ & $42(55.3)$ & 0.000 \\
\hline \multicolumn{5}{|l|}{ Organ or system dysfunction, $n(\%)$} \\
\hline Cardiovascular & 0 & $32(20.9)$ & $42(55.3)$ & 0.000 \\
\hline Respiratory & 0 & $58(37.9)$ & $2(2.6)$ & 0.000 \\
\hline Coagulation & 0 & $8(5.2)$ & $1(1.3)$ & 0.000 \\
\hline Electrolyte imbalance & $15(1.1)$ & $22(14.4)$ & $3(3.9)$ & 0.000 \\
\hline MODS & 0 & $27(17.6)$ & 0 & \\
\hline Deaths, $n(\%)$ & $1(0.1)$ & $53(34.6)$ & $1(1.3)$ & 0.000 \\
\hline
\end{tabular}

Group definitions: Sepsis, sepsis; SS, severe sepsis; SNS, SIRS-negative sepsis Definition of abbreviations: MODS, multiple organ dysfunction syndrome

a Values are taken from reference [15]; ${ }^{b}$ Numbers include 32 septic shock

fatality rate of $52.2 \%$ for coma (24/46) and $92.8 \%$ for deep coma (13/14) in the three groups. Most of the deaths occurred in SS except one each in S (aplastic anemia) and SNS. Infections of the lungs, central nervous system, and digestive system often led to SS or SNS. Nearly $90 \%$ SNS had respiratory tract infections (severe pneumonia), 55.3\% (42 cases) severe cardiovascular system dysfunction, and $8 \%$ (6 cases) severe encephalitis (including purulent meningitis and viral meningitis).

A total of 51 cases (1 in SNS, 50 in SS) was diagnosed as respiratory failure, but in 7 cases escalated treatment such as ventilator use was declined by parents. The ventilator-use time was $3.3 \pm 3.4$ [median (range) 2.0 [1-12]] days. Fifty-five patients of all sepsis died, of which 5 patients died during the 30-day follow-up period after discharge including one patient died of aplastic anemia, thus the mortality of sepsis was $3.4 \%$ (54/1606). Besides, 54 cases all died of the condition of severe sepsis (in SS and SNS group), thus the case specific mortality rate of severe sepsis was $23.6 \%(54 / 229)$, and 38 cases with severe sepsis $(70.3 \%, 38 / 54)$ died within three days from admission.

No evidence of specific variables for predicting organ dysfunction in SS and SNS from physical examination, or from the values of biochemical and cytological measurements, was found. The values of C-reactive protein in SNS were significantly lower than S and SS but skewed with very low average values (and median 14.4, range 0-390 for S, 4.2, 0.4-299 for SS and 1.6, 0.4-119 mg/100 mL for SNS), which also coincided with their clinical feature. The details in each group are compared in Table 2.

\section{Medications}

In $77.6 \%$ of all patients, antibiotics were given in the local level I and II hospitals and health care clinics 
Table 2 Medications in the three groups

\begin{tabular}{lllll}
\hline & Sepsis $^{\mathrm{c}}$ & SS $^{\mathrm{c}}$ & SNS & $p$ \\
\hline Cases, $n$ & 1377 & $153^{\mathrm{d}}$ & 76 & \\
Antimicrobial therapy & & & & \\
pre-hospital, $n$ (\%) & $1054(76.5)$ & $123(80.9)$ & $65(86.7)$ & 0.101 \\
during, n (\%) & $1354(98.3)$ & $148(96.7)$ & $76(100.0)$ & 0.177 \\
within 2 h, $n(\%)$ & $1243(91.7)$ & $132(88.6)$ & $69(90.8)$ & 0.422 \\
time, days mean \pm SD & $8.4 \pm 4.6$ & $10.3 \pm 7.9^{\mathrm{a}}$ & $12.4 \pm 5.5^{\mathrm{ab}}$ & 0.000 \\
Other therapy & & & & \\
GS, n (\%) & $500(36.4)$ & $120(79.5)^{\mathrm{a}}$ & $57(75.0)^{\mathrm{a}}$ & 0.000 \\
VA, $n(\%)$ & $23(0.5)$ & $103(67.3)^{\mathrm{a}}$ & $46(60.5)^{\mathrm{a}}$ & 0.000 \\
IA, $n$ (\%) & $3(0.2)$ & $92(60.1)^{\mathrm{a}}$ & $63(82.9)^{\mathrm{ab}}$ & 0.000 \\
Sedatives, $n(\%)$ & $100(7.3)$ & $109(71.2)^{\mathrm{a}}$ & $59(77.6)^{\mathrm{a}}$ & 0.000 \\
IVIG, $n(\%)$ & $52(3.8)$ & $49(32.2)^{\mathrm{a}}$ & $19(25.0)^{\mathrm{a}}$ & 0.000 \\
\hline
\end{tabular}

Group definition: Sepsis, sepsis; SS, severe sepsis; SNS, SIRS-negative sepsis Definition of abbreviations: pre-hospital, antimicrobial therapy was started prior to admission; during, antimicrobial therapy was given during hospitalization; within $2 \mathrm{~h}$, intravenous antimicrobial therapy was started within $2 \mathrm{~h}$ of recognizing sepsis; time, the entire period of antimicrobial treatment; GS, glucocorticosteroids; VA, vasoactive agents; IA, inotropes; IVIG, intravenous immunoglobulin

Statistical analysis: ${ }^{\mathrm{a}} p<0.01 \mathrm{vs}, \mathrm{S} ;{ }^{\mathrm{b}} p<0.01 \mathrm{vs}$, SS

${ }^{c}$ Values are taken from reference [15]; ${ }^{\mathrm{d}}$ Numbers include 32 septic shock

before admission, and $97.4 \%$ of which received in the first $2 \mathrm{~h}$ after admission, with $76.8 \%$ of the antibiotics treated as combined regimen. Cephalosporins and penicillins were the most frequently provided. Furthermore, significantly more patients in SS and SNS received antibiotics before admission $(P<0.001)$. The use of glucocorticosteroids was high, especially in SS (81.8\%) and SNS (71.1\%), and non-survivors had $79.3 \%$ treated with dexamethasone. The average duration of each course was $3.2 \pm 3.0$ (median (range) $2(1-19)$ ) days, in a range of dosage $0.1-0.3 \mathrm{mg} / \mathrm{kg} /$ day. In $11 \%$ of all patients received vasoactive drugs $(n=172)$, dopamine was the most frequently used $(n=142)$. Sixty-one percent $(n=106)$ of the survivors in SS and SNS groups received vasoactive drugs in contrast to $79.6 \%(n=43)$ of the non-survivors. Intravenous immunoglobulin was provided in 120 cases (7.5\% of all patients).

\section{Discussion}

In this parallel, multicenter prospective survey in Huai'an we found that the clinical features and outcomes of children presenting with SS and SNS share both diversity and similarities that warrants a uniform approach to diagnosis and treatment. SNS was mainly found in infants with infection of respiratory system and clinically manifested as cardiopulmonary and neurologic dysfunction. In contradiction to SS none of the SNS infants satisfied SIRS criteria. These differences may be caused by viral infection and/or under-developed immune system in response to the infection. However, no laboratory variables were found to identify SNS, or to judge response to therapy or predict outcome.

The international consensus criteria were entailed to early recognition of sepsis [12]. Early recognition and treatment of sepsis can decrease mortality $[19,20]$. However, the SIRS criteria have low specificity to identify infected patients at risk of exacerbation to severe sepsis or septic shock [22-24] and may miss one in eight adult patients with sepsis [13]. In our study, 76 (4.7\%) cases of SNS had normal temperature and WBC, and did not meet SIRS criteria for presumed sepsis. This may be similar to the group identified by Kaukonen and colleagues as having sepsis but not meeting the international consensus criteria [13], and provides further evidence that using the SIRS criteria for sepsis screening is suboptimal [13-16]. In addition, in resource limited environments the use of SIRS for the criteria of sepsis may result in about 33\% false-positives and has led to seeking alternatives to the traditional SIRS criteria in these settings [25]. We speculate that SIRS may be altered by response to medications such as antibiotics for a limited period, especially at level I clinics in this cohort (Table 2). However, if the SIRS persists, then clinical deterioration follows, leading to severe sepsis [26]. Patients in the SNS group had no history of SIRS, or any other explanation except sepsis for their clinical status, a phenomenon which has been previously reported [27]. It revealed that pediatric sepsis patient population may not share common clinical features and laboratory findings but SIRS-negative as a small proportion as those of adults [13, 14, 28].

The SS group was associated with a higher incidence of hypoxia and multiple organ dysfunction. In contrast in SNS, there were $90 \%$ as respiratory tract infections, $55.3 \%$ severe cardiovascular system dysfunction requiring vasopressors and inotropes, and 8\% severe encephalitis, though proportion of organ system dysfunction and deaths were lower (Table 1). The clinical courses were similar between SS and SNS, but their mechanisms related to organ system dysfunction and deaths are yet to be determined. The main implication of our findings is that the approach to management should be similar in children presenting with SS or SNS in light of the absence of SIRS criteria and no laboratory data to differentiate SS from SNS. While SIRS may be practically used for early screening for systemic infection in children, for the absence of SIRS criteria in young children, especially in infants, it does not preclude risks of developing SS or SNS.

Our study has limitations. Because there was no observation room in the local level I hospitals, outpatients with the possibility of developing sepsis may be missed because of an insufficient observation period. However, due to universal coverage of health insurance mandate, a 
robust regional transport system which also warranted prompt access to the level II and III hospitals, and the active admission policy and practice in the 11 participating hospitals, through regional infrastructure of pediatric emergency care, for the patients suspected of severe infection and/or organ dysfunction, few cases would be missed.

\section{Conclusion}

We found in a large sample of pediatric sepsis that a small group of patients who clinically manifested as implied, SIRS-negative, sepsis and were not identified using the international consensus criteria. These patients were infants predominantly with cardiopulmonary and neurologic dysfunction. Thus the findings of changes in mental status and cardiopulmonary morbidities in infants without SIRS criteria may indicate SNS and should prompt early intervention. Validation of our findings will rely on a larger cohort from other locations.

\section{Abbreviations}

CRP: C-Reactive protein; PICU: Pediatric intensive care unit; SIRS: Systemic inflammatory response syndrome; SNS: SIRS-Negative sepsis; SS: Severe sepsis; WBC: White blood cell counts

\section{Acknowledgements \\ List of institutions and investigators \\ The following hospitals and investigators participated in the Collaborative Study Group for Pediatric Sepsis in Huai'an: Children's Hospital of Fudan University, Shanghai (Bo Sun [study director], Yuanyuan Wang [coordinator]; Huai'an Maternity and Childrens' Hospital (Hongni Yue, Xiaofei Lin, Bing Li, Chunlin Yu); Huai'an First People's Hospital (Xiaochun Yang); Huai'an Second People's Hospital (Chunming Shan); Huaiyin District Hospital (Yujin Fan): Chuzhou District Hospital (Maotian Dong, Li Hao); Xuyi County Hospital (Yixing Zhang); Xuyi County Hospital for Traditional Chinese Medicine (Wenlong Lin); Lianshui County Hospital (Xiaofeng Zuo, Tingting Yin): Lianshui County Hospital for Traditional Chinese Medicine (Ping Su); Jinhu County Hospital (Yongbo Heng); Hongze County Hospital (Jinzhong Xu).}

\section{Funding}

Supported by grants from Shanghai Municipal Department of Health (No. LJ06038), and Chinese Medical Board of New York (No. 03-786). The funding bodies had no influence on the design of the study, collection, analysis, and interpretation of data; and in writing the manuscript.

\section{Availability of data and materials}

The datasets used and/or analysed during the current study are available from the corresponding author on reasonable request.

\section{Authors' contributions}

IW conceptualized, executed the study protocol, coordinated with coinvestigators from the participating centers, collected and analyzed all the data, drafted the manuscript and approved its final version. XL participated as coinvestigator, contributed to the whole study by providing clinical data file from his unit as a core part in the whole database, participated in co-ordination, data collection and analysis, appraised and approved the final version. $\mathrm{HY}$ in part designed and conceptualized the study, coordinated the regional network to have accomplished the collaboration, revised and approved the final version. NK in part designed and conceptualized the study, participated in the staff training program, edited the manuscript and approved its final version. BS designed and conceptualized the study, interpreted the data analysis, edited the manuscript and approved its final version.

\section{Ethics approval and consent to participate}

The ethics committee of Children's Hospital of Fudan University approved the study design and protocol, and this approval was adopted by all the participating centers in Huai'an through respective institutional review boards/scientific committees in accordance with the Chinese regulations for clinical investigation. As no specific intervention was involved in the study protocol, informed consent was waived.

\section{Consent for publication}

Not applicable.

\section{Competing interests}

All the authors have declared no any financial and non-financial competing interests to disclose.

\section{Publisher's Note}

Springer Nature remains neutral with regard to jurisdictional claims in published maps and institutional affiliations.

\section{Author details}

'Departments of Pediatrics and Pediatric Critical Care, Children's Hospital of Fudan University, 399 Wanyuan Road, Shanghai 201102, China. 'Departments of Pediatrics and Pediatric Critical Care, Huai'an Women and Children's Hospital, Huai'an 223002, Jiangsu, China. ${ }^{3}$ Department of Pediatrics, University of British-Columbia and BC Children's Hospital, Vancouver, BC, Canada.

Received: 28 May 2018 Accepted: 4 December 2018 Published online: 08 January 2019

References

1. Kissoon N, Carcillo JA, Espinosa V, et al. World Federation of Pediatric Intensive Care and Critical Care Societies: Global sepsis initiative. Pediatr Crit Care Med. 2011;12:494-503.

2. Black RE, Cousens S, Johnson HL, et al. Global, regional, and national causes of child mortality in 2008: a systematic analysis. Lancet. 2010;375:1969-87.

3. Mangia CMF, Kissoon N, Carcillo JA. Sepsis and septic shock: a global overview. J Pediatr Infect Dis. 2009;4:71-6.

4. Watson RS, Carcillo JA, Linde-Zwirble WT, et al. The epidemiology of severe sepsis in children in the United States. Am J Respir Crit Care Med. 2003;167:695-701.

5. Inwald DP, Tasker RC, Peters MJ, et al. Emergency management of children with severe sepsis in the United Kingdom: the results of the Paediatric Intensive Care Society sepsis audit. Arch Dis Child. 2009;94(5):348-53.

6. De Oliveira CF, de Oliveira DS, Gottschald AF, et al. ACCM/PALS haemodynamic support guidelines for paediatric septic shock: an outcomes comparison with and without monitoring central venous oxygen saturation. Intensive Care Med. 2008:34:1065-75.

7. Branco RG, Garcia PC, Piva JP, et al. Glucose level and risk of mortality in pediatric septic shock. Pediatr Crit Care Med. 2005;6:470-2.

8. Collaborative Group for the Study of Sepsis in PICUs in Beijing Area. Clinical study on sepsis in 2 pediatric intensive care units in Beijing. Zhonghua Er Ke Za Zhi. Chin J Pediatr. 2012;50:178-83.

9. $Y u$ W, Lu Z, Wang Y, et al. The epidemiology of acute respiratory distress syndrome in pediatric intensive care units in China. Intensive Care Med. 2009;35:136-43.

10. Hu X, Qian S, Xu F, et al. Acute hypoxemic respiratory failure and acute respiratory distress syndrome in pediatric intensive care units in China. Acta Paediatr. 2010;99:715-21.

11. Zhu $Y, X u F, L u X$, et al. Mortality and morbidity of acute hypoxemic respiratory failure and acute respiratory distress syndrome in infants and young children. Chin Med J. 2012;125:2265-71.

12. Goldstein B, Giroir B, Randolph A. International pediatric sepsis consensus conference: definitions for sepsis and organ dysfunction in pediatrics. Pediatr Crit Care Med. 2005;6:2-8.

13. Kaukonen KM, Bailey M, Pilcher D, et al. Systemic inflammatory response syndrome criteria in defining severe sepsis. N Engl J Med. 2015;372:1629-38.

14. Ruth A, McCracken CE, Fortenberry JD, et al. Pediatric severe sepsis: current trends and outcomes from the Pediatric Health Information Systems database. Pediatr Crit Care Med. 2014;15:828-38.

15. Wang $Y$, Sun $B$, Yue $H$, et al. An epidemiologic survey of pediatric sepsis in regional hospitals in China. Pediatr Crit Care Med. 2014;15:814-20.

16. Pavare J, Grope I, Gardovska D. Prevalence of systemic inflammatory response syndrome (SIRS) in hospitalized children: a point prevalence study. BMC Pediatr. 2009;9:25. 
17. Singhi S, Khilnani P, Lodha R, et al. Guidelines for treatment of septic shock in resource limited environments. J Pediatr Infect Dis. 2009;4:173-92.

18. Carcillo JA, Fields Al. Clinical practice parameters for hemodynamic support of pediatric and neonatal patients in septic shock. Crit Care Med. 2002;30:1365-78.

19. Dunser MW, Festic E, Dondorp A, et al. Recommendations for sepsis management in resource-limited settings. Intensive Care Med. 2012;38:557-74.

20. Parker MM. Pediatric definitions for sepsis: it's about time. Pediatr Crit Care Med. 2005;6:83-4

21. Wiwanitkit V. Extreme thrombocytosis: what are the etiologies? Clin Appl Thromb Hemost. 2006;12:85-7.

22. Dremsizov T, Clermont G, Kellum JA, et al. Severe sepsis in communityacquired pneumonia: when does it happen, and do systemic inflammatory response syndrome criteria help predict course ? Chest. 2006;129:968-78.

23. Claessens YE, Dhainaut JF. Diagnosis and treatment of severe sepsis. Crit Care. 2007;1 1(Suppl 5):S2.

24. Alberti C, Brun-Buisson C, Chevret $\mathrm{S}$, et al. Systemic inflammatory response and progression to severe sepsis in critically ill infected patients. Am J Respir Crit Care Med. 2005;171:461-8.

25. Wiens MO, Kumbakumba E, Kissoon N, et al. Pediatric sepsis in the developing world: challenges in defining sepsis and issues in post-discharge mortality. Clin Epidemiol. 2012:4:319-25.

26. Weiss SL, Parker B, Bullock ME, et al. Defining pediatric sepsis by different criteria: discrepancies in populations and implications for clinical practice. Pediatr Crit Care Med. 2012;13:e219-26.

27. Kuong EE. To M, Yuen MH, et al. Pitfalls in diagnosing septic arthritis in Hong Kong children: ten years' experience. Hong Kong Med J. 2012;18(6):482-7.

28. Dellinger RP, Levy MM, Carlet JM, et al. Surviving Sepsis campaign: international guidelines for management of severe sepsis and septic shock: 2008. Intens Care Med. 2008:34:17-60

Ready to submit your research? Choose BMC and benefit from:

- fast, convenient online submission

- thorough peer review by experienced researchers in your field

- rapid publication on acceptance

- support for research data, including large and complex data types

- gold Open Access which fosters wider collaboration and increased citations

- maximum visibility for your research: over $100 \mathrm{M}$ website views per year

At $\mathrm{BMC}$, research is always in progress.

Learn more biomedcentral.com/submissions 